\title{
Tensor correlations in nuclei and exclusive electron scattering
}

\author{
J. Ryckebusch, ${ }^{*}$ S. Janssen, W. Van Nespen, and D. Debruyne \\ Department of Subatomic and Radiation Physics, University of Gent, Proeftuinstraat 86, B-9000 Gent, Belgium
}

(Received 19 August 1999; published 18 January 2000)

\begin{abstract}
The effect of tensor nucleon-nucleon correlations upon exclusive and semiexclusive electronuclear reactions is studied. Differential cross sections for the semiexclusive ${ }^{16} \mathrm{O}\left(e, e^{\prime} p\right)$ and exclusive ${ }^{16} \mathrm{O}\left(e, e^{\prime} p n\right)$ processes are computed by explicitly evaluating the dynamical electromagnetic coupling to a tensor correlated nucleon pair. In both reaction channels the tensor correlations contribute in a very substantial way. Tensor correlations are found to generate more electronuclear strength than central Jastrow correlations do.
\end{abstract}

PACS number(s): 25.30.-c, 21.30.Fe, 24.10.-i

In the history of nuclear physics, it has been notoriously difficult to detect signals that directly point towards phenomena beyond the scope of the effective mean-field theories. This holds, in particular, for the short-range correlations that reflect the remnants of the hard-core part of the nucleonnucleon $(N N)$ force in the medium. Recently, manifestations for strongly correlated proton pairs and the existence of Jastrow-like correlations emerged from the simultaneous detection of two protons upon absorption of one (virtual) photon by an atomic nucleus [1,2]. Nuclear many-body theories have produced vastly different predictions for the short-range behavior of nuclei. The ongoing exclusive $A\left(e, e^{\prime} p p\right)$ studies are expected to provide stringent tests of these theories. The correlations probed in proton-proton knockout are predominantly the state-independent scalar ones (often referred to as Jastrow correlations) related to the hard core part of the $N N$ force. The tensor force, which is operative at intermediate internucleon distances $(\approx 1-2.5 \mathrm{fm})$, is established to be an important ingredient of the $N N$ force in the medium and is believed to be another source of important $N N$ correlations which go beyond the mean-field level [3]. High momentum components in the deuteron wave function, for example, are ascribed to a $D$-state admixture and are a direct manifestation of the presence of tensor correlations in the proton-neutron system. For a long time, the tensor interaction has been established to be a rather weak but essential ingredient of the effective $N N$ force. Despite intensive research a recent review [4] quoted its role as "elusive." Earlier studies of the role played by tensor correlations in electron scattering concentrated on inclusive $A\left(e, e^{\prime}\right)$ response functions, for which there are many competing effects and unambiguous information on the tensor correlations, might be difficult to extract $[5,6]$. In the near future, exclusive experiments that aim at probing both the proton-proton and proton-neutron correlations will be performed at MAMI and TJNAF. At MAMI, where central short-range correlations in nuclei are being studied with the aid of the $A\left(e, e^{\prime} p p\right)$ reaction [2,7], highresolution $A\left(e, e^{\prime} p n\right)$ measurements have been scheduled for the target nuclei ${ }^{3} \mathrm{He}[8]$ and ${ }^{16} \mathrm{O}$ [9]. These measurements will be performed at four-momentum transfers of the order $Q^{2} \approx 0.05(\mathrm{GeV} / c)^{2}$. At TJNAF, on the other hand, the small distance structure of nuclei will be probed with the aid of the ${ }^{12} \mathrm{C}\left(e, e^{\prime} p N\right)(N=p, n)$ reaction at $Q^{2} \geqslant 2(\mathrm{GeV} / c)^{2}$

\footnotetext{
*Electronic address: jan.ryckebusch@rug.ac.be
}

[10]. In the light of the upcoming experiments, we present model calculations that aim at exploring the possibility of using exclusive and semiexclusive electronuclear reactions to elucidate the role of tensor correlations in the nuclear medium.

To establish a connection between the $N N$ correlations and the measured electronuclear cross sections, we remark that the response of the target nucleus (with "correlated" ground-state wave function $\left|\overline{\Psi_{i}}\right\rangle$ ) to the electromagnetic probe is determined by matrix elements of the form

$$
\frac{\left\langle\overline{\Psi_{f}}\left|J_{\mu}(q)\right| \overline{\Psi_{i}}\right\rangle}{\left\langle\overline{\Psi_{f}} \mid \overline{\Psi_{f}}\right\rangle\left\langle\overline{\Psi_{i}} \mid \overline{\Psi_{i}}\right\rangle} .
$$

We introduce "correlated" nuclear wave functions in a standard manner,

$$
|\bar{\Psi}\rangle=\hat{\mathcal{S}}\left[\prod_{i<j=1}^{A}\left(1-g_{c}\left(r_{i j}\right)+f_{t \tau}\left(r_{i j}\right) \widehat{S_{i j}} \vec{\tau}_{i} \cdot \vec{\tau}_{j}\right)\right]|\Psi\rangle,
$$

where $|\Psi\rangle$ is the uncorrelated and normalized (Slater determinant) wave function obtained from a mean-field calculation, $\hat{\mathcal{S}}$ is the symmetrizer, $g_{c}$ is the central correlation function that accounts for (state-independent or Jastrow) shortrange effects, and $\widehat{S_{i j}}$ is the tensor operator that introduces state-dependent correlations for the $S=1$ components in the nuclear wave functions. Strictly speaking, the correlation operator also contains spin-isospin and spin-orbit terms. The $g_{c}$ and $f_{t \tau}$ are, however, the most important ones and without their presence finite nuclei would simply be unbound [3]. A striking feature of the correlation functions $g_{c}$ and $f_{t \tau}$ is that they exhibit a modest $A$ dependence [3]. This makes them a universal feature of atomic nuclei whose experimental determination is of the utmost importance. The hadronic current operator $J_{\mu}(q)$ in the above expression accounts for the coupling of the electromagnetic field to the nuclear system. In our calculations, the one-body part $\left(J_{\mu}^{[1]}\right)$ has been implemented in the standard impulse approximation fashion, whereas the two-body currents $\left(J_{\mu}^{[2]}\right)$ include the conventional pion-exchange and intermediate $\Delta_{33}$-resonance terms [11]. The matrix element of Eq. (1) contains 2,3, . . A-body correlations and is usually calculated with the aid of an expansion that is cut at some order. It is worth stressing that most of the cluster expansions for correlated systems that are outlined in the literature refer to calculations addressing the 
"inclusive" response of the correlated target nucleus to the electromagnetic probe. Hereby, closure properties can be exploited to help reducing the complexity of the calculations. In the $\left(e, e^{\prime} p\right)$ case, for example, closure can be applied when integrating over all excitation energies of the residual $A-1$ system [12]. This quantity, however, is experimentally not accessible as it would involve integrations over kinematics regions where the measured $\left(e, e^{\prime} p\right)$ strength is contaminated by pion production [13]. Here we aim at computing the effect of the correlations on some well-defined parts of the phase space in exclusive and semi-exclusive electronuclear processes. Accordingly, no closure relations can be applied and most of the known cluster expansion techniques are not directly applicable. In inclusive ${ }^{12} \mathrm{C}\left(e, e^{\prime}\right)$ [14] and ${ }^{4} \mathrm{He}\left(e, e^{\prime} d\right)[15]$ calculations the lowest-order approximation (LOA) was observed to account for the major $N N$ correlations effects. Given that exclusive $A\left(e, e^{\prime} N N\right)$ processes are confined to two-nucleon phase space, three- and higherbody "correlations" are expected to produce even smaller corrections than in the inclusive case. For that reason, the transition matrix element of Eq. (1) was evaluated in the LOA. This procedure results in a clear separation between the contributions from the uncorrelated mean-field wave function that reads

$$
\left\langle\Psi_{f}\left|\left(\sum_{i=1}^{A} J_{\mu}^{[1]}(i ; q)+\sum_{i<j=1}^{A} J_{\mu}^{[2]}(i, j ; q)\right)\right| \Psi_{i}\right\rangle,
$$

and the ones that can be unambiguously attributed to the $N N$ correlations,

$$
\begin{array}{r}
\left\langle\Psi_{f}\right| \sum_{i<j=1}^{A}\left[\left(J_{\mu}^{[1]}(i ; q)+J_{\mu}^{[1]}(j ; q)+J_{\mu}^{[2]}(i, j ; q)\right)\right. \\
\left.\quad \times\left(-g_{c}\left(r_{i j}\right)+f_{t \tau}\left(r_{i j}\right) \widehat{S_{i j}} \vec{\tau}_{i} \cdot \vec{\tau}_{j}\right)+\text { H.c. }\right]\left|\Psi_{i}\right\rangle .
\end{array}
$$

The correlation functions $g_{c}$ and $f_{t \tau}$ establish the link between the measurements and the nuclear many-body theories, and contain the information of the "beyond meanfield'" structure of nuclei. In inclusive $A\left(e, e^{\prime}\right)$ and exclusive $A\left(e, e^{\prime} p\right)$ reactions, signals from the "correlation", part (4) in the transition matrix elements are frequently obscured by the dominant one-body term $J_{\mu}^{[1]}$ in Eq. (3). For uncorrelated wave functions, two-nucleon knockout strength can solely be generated by the one-body current through final-state interaction effects, e.g., $A\left(e, e^{\prime} p\right) A-1$ followed by $A$ $-1\left(p, p^{\prime} n\right)$. We will consider kinematical regimes in which the contaminant effect of rescattering processes is minimized. To that purpose we compute proton neutron knockout processes from a light nucleus $\left({ }^{16} \mathrm{O}\right)$ with the condition that the residual nucleus $\left({ }^{14} \mathrm{~N}\right)$ is created at low excitation energies. Moreover, we will consider the situation that the two nucleons are ejected along the momentum transfer ("superparallel kinematics"). Then, $A-1\left(p, p^{\prime} n\right)$ rescattering processes involve large momentum transfers and are heavily suppressed. This feature of heavily suppressed one-body cur- rent contributions to the uncorrelated matrix element of Eq. (3), opens up good opportunities to acquire a precise understanding of $N N$ correlations by studying two-nucleon knockout processes.

Direct information on the precise role of tensor correlations in nuclei is expected from triple coincidence $A\left(e, e^{\prime} p n\right)$ experiments, provided that one can separate the signals from the electromagnetic coupling to a tensor correlated proton-neutron pair from two-body current contributions. In $A\left(e, e^{\prime} p p\right)$ and $A\left(e, e^{\prime} p n\right)$ studies, a powerful tool in the search for $N N$ correlations is the selectivity of the final state with respect to the quantum numbers of the correlated nucleon pair that actively participates in the reaction process $[16,17,19]$. We have calculated ${ }^{16} \mathrm{O}\left(e, e^{\prime} p n\right){ }^{14} \mathrm{~N}$ cross sections for excitation of specific states in ${ }^{14} \mathrm{~N}$. The results are displayed in Fig. 1 and include apart from the central and tensor correlations, the "uncorrelated" contribution of competing meson-exchange and intermediate $\Delta$-resonance twobody current contributions. The model employed to calculate these cross sections includes a distorted wave description of the ejectiles and was extensively discussed in Ref. [11]. Restricting ourselves to the (dominant) $p$-shell components, the following selectivity for the quantum numbers of the active proton-neutron pair emerges when considering ${ }^{16} \mathrm{O}\left(e, e^{\prime} p n\right)$ decay to the different angular momentum states in ${ }^{14} \mathrm{~N}$

$$
\begin{gathered}
J_{R}=0+{ }^{+1} S_{0}(\Lambda=0),{ }^{3} P_{1}(\Lambda=1), \\
J_{R}=1{ }^{+3} S_{1}(\Lambda=0,2),{ }^{3} P_{0,1,2}(\Lambda=1), \\
{ }^{1} P_{1}(\Lambda=1),{ }^{3} D_{1}(\Lambda=0), \\
J_{R}=2{ }^{+1} S_{0}(\Lambda=2),{ }^{3} S_{1}(\Lambda=0,2),{ }^{3} P_{1,2}(\Lambda=1), \\
{ }^{1} D_{2}(\Lambda=0),{ }^{3} D_{2}(\Lambda=0),
\end{gathered}
$$

where the standard convention $\left({ }^{2 S+1} L_{J}\right)$ for the relative twonucleon wave function is adopted and $\Lambda$ is the orbital quantum number corresponding with the center-of-mass (COM) motion of the pair. We have calculated the differential cross section for these states in the low-energy spectrum of ${ }^{14} \mathrm{~N}$ that are established to have a two-hole character relative to the ground state of ${ }^{16} \mathrm{O}[20]$ and are therefore expected to be strongly populated in a direct ${ }^{16} \mathrm{O}\left(e, e^{\prime} p n\right)$ reaction [17]. The two-body overlap amplitudes employed in our calculations are from Ref. [21]. The $g_{c}$ and $f_{t \tau}$ correlation functions are those from [22]. They are obtained in variational calculations for the ${ }^{16} \mathrm{O}$ ground state with the Argonne $\mathrm{v}_{14} \mathrm{NN}$ potential. We have selected so-called "super-parallel"' kinematics which makes the two nucleons to move along the momentum transfer. It has become customary in the discussion of two-nucleon knockout processes to present the results in terms of the pair missing momentum. In a spectator model the pair missing momentum coincides with the inital COM momentum of the ejected nucleon pair. As inferrred from the missing-momentum dependence of the cross sections contained in Fig. 1, the ground state has a mixed $\Lambda=0,2$ character, whereas the $J_{R}=1^{+}$state at $E_{x}=3.95 \mathrm{MeV}$ reflects a $\Lambda=0$ shape. As evidenced by the results of Fig. 1 the effect 

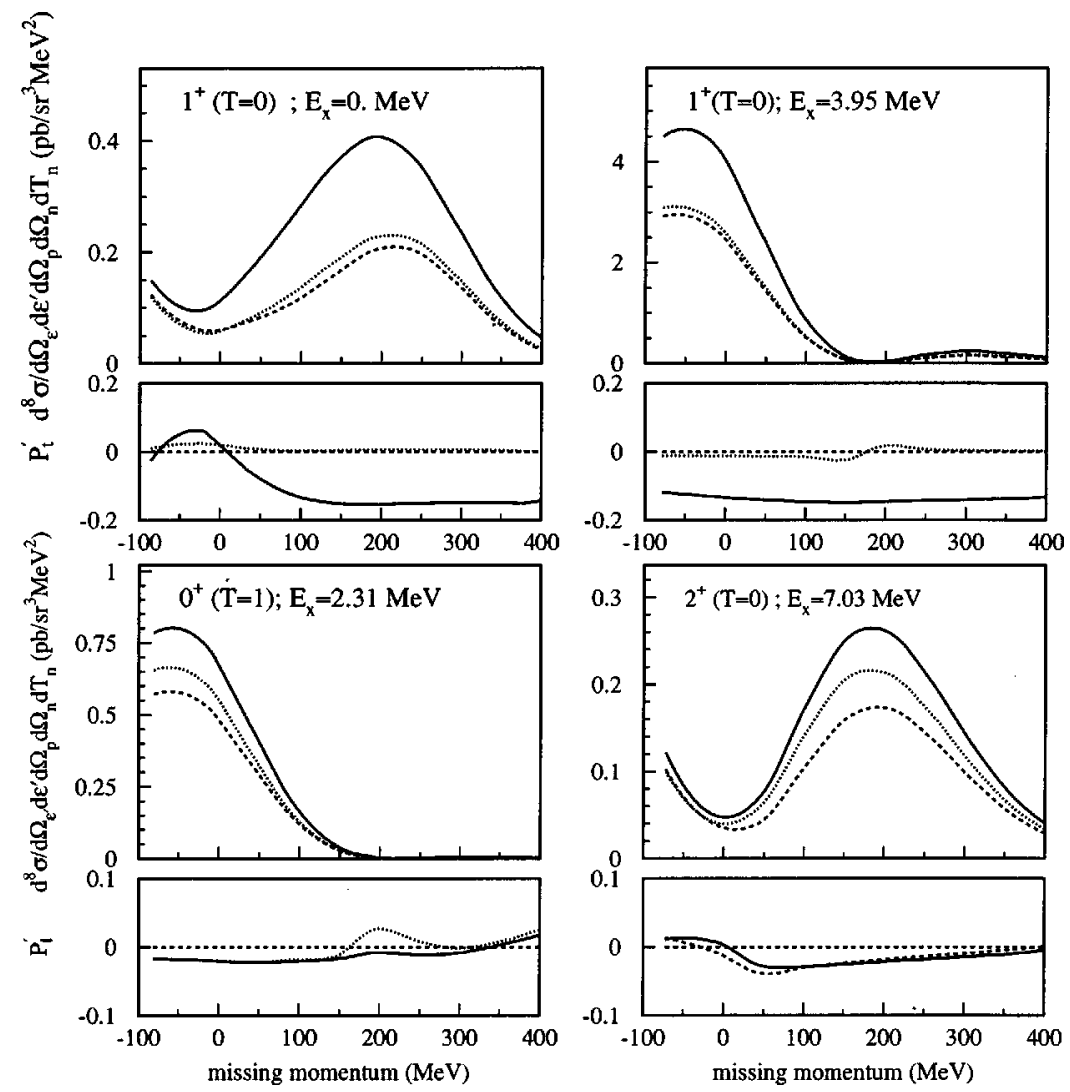

FIG. 1. The missing momentum dependence of the ${ }^{16} \mathrm{O}\left(e, e^{\prime} p n\right)$ differential cross section and recoil polarization observable $P_{t}^{\prime}$ for excitation of the low-lying two-hole states in ${ }^{14} \mathrm{~N}$ at $\epsilon=855 \mathrm{MeV}, \epsilon^{\prime}=640 \mathrm{MeV}$, and $\theta_{e^{\prime}}=18^{\circ}$. The calculations refer to the situation in which the proton (neutron) is detected parallel (antiparallel) to the direction of the momentum transfer. The solid curve is calculated in the distorted-wave approximation including the two-body currents, central, and tensor correlations. The dashed line is the contribution from the two-body currents. The dotted lines omit tensor correlations from the full calculation.

of the tensor correlations in exclusive proton-neutron knockout is large. Neglecting the tensor correlations would result in a cross section for the ground-state transition that is about a factor of 2 too small. The strongest sensitivity to the tensor correlations, though, is found in the peak of the cross sections for the transition to the $1^{+}$states at, respectively, $E_{x}$ $=0$ and $3.95 \mathrm{MeV}$. As is clear from the pair combinations contained in Eq. (5) this enhanced sensitivity to the tensor correlations corresponds with the situation that the reaction is dominated by absorption on proton-neutron pairs in a ${ }^{3} S_{1}$ configuration. The predicted effect of the central correlations is small in comparison with the tensor contributions. For the transition to the $0^{+}$and $2^{+}$states, the effect of the central and tensor correlations is about of equal importance and relatively modest. This can be explained by remarking that for these states the ${ }^{3} S_{1}$ configuration does not $\left(0^{+}\right)$or only marginally contribute $\left(2^{+}\right)$. A striking feature is the big difference in the computed differential cross sections for the two $1^{+}$states. A detailed ${ }^{16} \mathrm{O}\left(\pi^{+}, p p\right){ }^{14} \mathrm{~N}$ measurement reported in Ref. [18] determined that the transition cross section has a mixed $\Lambda=0,2$ for the ground state and a predominant $\Lambda=0$ character for the $E_{x}=3.95 \mathrm{MeV}$ state. Moreover, the differential cross section for the $E_{x}=3.95 \mathrm{MeV}$ state was determined to be substantially larger than for the ground state. In many respects the $\left(\pi^{+}, p p\right)$ process bears resemblance with the $\left(e, e^{\prime} p n\right)$ reaction and our computed ${ }^{16} \mathrm{O}\left(e, e^{\prime} p n\right)$ differential cross sections for the $1^{+}$states exhibit exactly the same qualitative features than those observed in the ${ }^{16} \mathrm{O}\left(\pi^{+}, p p\right){ }^{14} \mathrm{~N}$ data of Ref. [18].

Also shown in Fig. 1 are the predictions for the double polarization observables $P_{t}^{\prime}$. This variable can be determined in $A\left(\vec{e}, e^{\prime} \vec{p} n\right)$ measurements and determines the recoil polarization along the direction $\hat{t}$ which is in the reaction plane orthogonal to the direction of ejected proton's momentum. In superparallel kinematics, $P_{t}^{\prime}$ is uniquely determined by the $W_{L T}^{\prime}$ structure function. The background of two-body current contributions to proton-neutron knockout is almost exclusively transverse. With no ground-state correlations contributing, $P_{t}^{\prime}$ is extremely small. For these transitions with strong contributions from the tensor correlations, the calculations predict large values $P_{t}^{\prime}$, though. In that respect, the recoil polarization observable is a measure for the importance of tensor correlations in the medium. Double polarization observables have been frequently shown to be relatively free of ambiguities with respect to the final-state interaction [19].

In general, triple coincidence measurements are challenging. An indirect way of accessing the ground-state correla- 
tions is the semiexclusive $A\left(e, e^{\prime} p\right)$ process. Indeed, when probing higher missing energies $E_{m}=\omega-T_{p}-T_{A-1}$, the residual nucleus is created at a high excitation energy $\left(E_{x}\right.$ $\gtrsim 30 \mathrm{MeV}$ ). In this regime, exclusive single-nucleon knockout through the "uncorrelated" one-body current operator of Eq. (3) is heavily suppressed and besides the mesonic degrees of freedom, the "correlations" terms in Eq. (4) are expected to feed the $A\left(e, e^{\prime} p\right)$ channel. As the "correlations" are predominantly of two-body nature, the "correlated" $A\left(e, e^{\prime} p\right)$ strength will manifest itself as two-nucleon knockout. Often, in interpreting semiexclusive $A\left(e, e^{\prime} p\right)$ and inclusive $A\left(e, e^{\prime}\right)$ processes, a factorization scheme is adopted. Hereby, the hadronic part of the $A\left(e, e^{\prime} p\right)$ cross section is written in terms of the probability to find a nucleon with a certain momentum for a fixed missing energy of the $A-1$ spectators (the so-called spectral function). For the results presented here, an unfactorized approach is adopted. The method was outlined in Ref. [11] and is based on explicitly calculating the contribution from the operator $\left(J_{\mu}^{[1]}(i ; q)+J_{\mu}^{[1]}(j ; q)\right)\left(-g_{c}\left(r_{i j}\right)+f_{t \tau}\left(r_{i j}\right) \widehat{S_{i j}} \vec{\tau}_{i} \cdot \vec{\tau}_{j}\right)$ to the two-nucleon knockout channels $\left[A\left(e, e^{\prime} p n\right)\right]$ and $\left.A\left(e, e^{\prime} p p\right)\right]$ and integrating over the complete phase space of the undetected nucleon. The calculated missing energy spectrum of the ${ }^{16} \mathrm{O}\left(e, e^{\prime} p\right)$ reaction at $\omega=300 \mathrm{MeV}$ and $|\vec{q}|=416 \mathrm{MeV} / c$ is shown in Fig. 2 for a number of proton angles. It is worth stressing that in the absence of $N N$ correlations all computed strengths in Fig. 2 would vanish identically. As the angle $\theta_{p q}$, which is the polar angle of the detected proton relative to the direction of the momentum transfer, grows higher initial proton momenta are probed. A striking feature of the results is that the tensor correlations generate a few times more "correlated" ${ }^{16} \mathrm{O}\left(e, e^{\prime} p\right)$ strength than the central short-range correlations do. At $\theta_{p q}$ $=40^{\circ}$, where the missing (or, initial proton) momentum ranges over $275 \leqslant p_{m} \leqslant 450 \mathrm{MeV} / c$, inclusion of the tensor correlations increases the strength with a factor of 5 . As clearly illustrated in Fig. 2, most of the "correlated" $\left(e, e^{\prime} p\right)$ strength can be ascribed to the proton-neutron knockout channel. With increasing polar angle $\theta_{p q}$, higher missing momenta are probed and the central correlations gain in relative importance. This can be explained by considering that central correlations refer to the hard part of the $N N$ force, whereas tensor correlations are of somewhat larger range ( $1 \lesssim r \lessgtr 3 \mathrm{fm}$ ). Qualitatively, the central and tensor correlations exhibit a similar missing energy behavior for all polar angles included in Fig. 2. This feature reflects the fact that both the central and tensor correlations are mostly affecting nucleon pairs in relative $S$ states, thus imposing stringent kinematical constraints on the $\left(E_{m}, p_{m}\right)$ regions where the strength attributed to the $N N$ correlations resides [24].

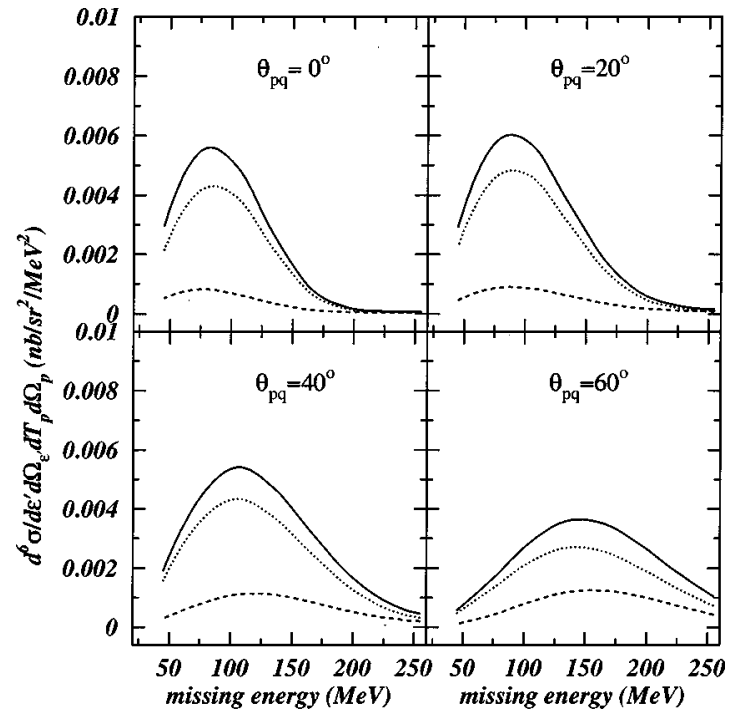

FIG. 2. The missing-energy dependence of the computed contribution from $N N$ correlations to the semiexclusive ${ }^{16} \mathrm{O}\left(e, e^{\prime} p\right)$ differential cross section at various proton polar angles. Dashed curves include only the central correlations; solid curves, both central and tensor correlations. The dotted curve is the calculated contribution from $\left(e, e^{\prime} p n\right)$ including tensor and central correlations. The electron kinematics is determined by $\epsilon=1.2 \mathrm{GeV}, \epsilon^{\prime}=0.9$ $\mathrm{GeV}$, and $\theta_{e}=16^{\circ}$.

Summarizing, we have presented a framework permitting a systematic investigation of the effects of state-independent (central) and state-dependent (tensor) correlations upon $A\left(e, e^{\prime} N N\right)$ and semiexclusive $A\left(e, e^{\prime} p\right)$ differential cross sections. The calculations reveal that the sensitivity of the ${ }^{16} \mathrm{O}\left(e, e^{\prime} p n\right)$ cross sections to central correlations is rather modest. On the contrary, strong signals from the tensor correlations appear in these regions of the $d \Omega_{p} d E_{p} d \Omega_{n} d E_{n}$ phase space whenever the inital photoabsorption happens to occur on ${ }^{3} S_{1}$ proton-neutron pairs. The tensor correlations are further predicted to produce substantially more semiexclusive ${ }^{16} \mathrm{O}\left(e, e^{\prime} p\right)$ strength than the central (Jastrow) correlations do. This peculiar feature of how the $N N$ correlations manifest themselves in electronuclear processes, makes the proton-neutron knockout channel dominate the semiexclusive $\left(e, e^{\prime} p\right)$ strength and could be considered as a microscopic confirmation of the suggested quasideuteron scaling of the "correlated" $A\left(e, e^{\prime}\right)$ strength [23]. Concluding, our results indicate that exclusive electronuclear reactions are a promising tool for providing insight into the role of tensor correlations in the nuclear medium.

This work was supported by the Fund for Scientific Research of Flanders under Contract No. 4.0061.99 and the University Research Council.
[1] C.J.G. Onderwater et al., Phys. Rev. Lett. 81, 2213 (1998).

[2] K.I. Blomqvist et al., Phys. Lett. B 421, 71 (1998).

[3] O. Benhar and V.R. Pandharipande, Rev. Mod. Phys. 65, 817
(1993).

[4] M.S. Fayache, L. Zamick, and B. Castel, Phys. Rep. 290, 201 (1997). 
[5] G. Orlandini, M. Traini, and F. Delagiacoma, Nuovo Cimento A 76, 246 (1983).

[6] A. Fabrocini, Phys. Rev. C 55, 338 (1997).

[7] G. Rosner, in Proceedings of the Conference on Perspectives in Hadronic Physics, ICTP, Trieste, Italy, 1997, edited by S. Boffi, C. Ciofi degli Atti, and M.M. Gianninni (World Scientific, Singapore, 1998), p. 185.

[8] Investigation of the ${ }^{3} \mathrm{He}\left(e, e^{\prime} p n\right)$ reaction (Contact persons $\mathrm{E}$. Jans, T. Hehl and G. Rosner); experiment to be performed in the A1 Hall at MAMI.

[9] First Study of the ${ }^{16} \mathrm{O}\left(e, e^{\prime} p n\right)$ reaction (Contact persons $\mathrm{P}$. Grabmayr and G. Rosner); experiment to be performed in the A1 Hall at MAMI.

[10] TJNAF experiment E97-106 "Studying the Internal Smalldistance Structure of Nuclei via the Triple Coincidence $\left(e, e^{\prime} p+N\right)$ measurement'" (spokesperson E. Piasetzky).

[11] J. Ryckebusch, V. Van der Sluys, K. Heyde, H. Holvoet, W. Van Nespen, M. Waroquier, and M. Vanderhaeghen, Nucl. Phys. A624, 581 (1997).

[12] H. Morita, C. Ciofi degli Atti, and D. Treleani, Phys. Rev. C 60, 034603 (1999).
[13] A. Gil, J. Nieves, and E. Oset, Nucl. Phys. A627, 599 (1997).

[14] Giampaolo Có and Antonio M. Lallena, Phys. Rev. C 57, 145 (1998).

[15] W. Leidemann, G. Orlandini, M. Traini, and E.L. Tomusiak, Phys. Rev. C 50, 630 (1994).

[16] G. Giusti, F.D. Pacati, K. Allaart, W. Geurts, H. Müther, and W. Dickhoff, Phys. Rev. C 57, 1691 (1998).

[17] C. Giusti, H. Müther, F.D. Pacati, and M. Stauf, Phys. Rev. C 60, 054608 (1999).

[18] R.A. Schumacher et al., Phys. Rev. C 38, 2205 (1988).

[19] Jan Ryckebusch, Wim Van Nespen, and Dimitri Debruyne, Phys. Lett. B 441, 1 (1998).

[20] J.L. Snelgrove and E. Kashy, Phys. Rev. 187, 1259 (1969).

[21] S. Cohen and D. Kurath, Nucl. Phys. A141, 145 (1970).

[22] S.C. Pieper, R.B. Wiringa, and V.R. Pandharipande, Phys. Rev. C 46, 1741 (1992).

[23] L.L. Frankfurt, M.I. Strikman, D.B. Day, and M. Sargsyan, Phys. Rev. C 48, 2451 (1993).

[24] C. Ciofi degli Atti and S. Simula, Phys. Rev. C 53, 1689 (1996). 